Pacific

Journal of

Mathematics

INDEX THEORY OF TOEPLITZ OPERATORS ASSOCIATED TO TRANSFORMATION GROUP $C^{*}$-ALGEBRAS

\title{
EFTON PARK
}




\title{
INDEX THEORY OF TOEPLITZ OPERATORS ASSOCIATED TO TRANSFORMATION GROUP $C^{*}$-ALGEBRAS
}

\author{
EFTON PARK
}

\begin{abstract}
Let $\Gamma$ be a finite discrete group acting smoothly on a compact manifold $X$, and let $D$ be a first-order elliptic self-adjoint $\Gamma$-equivariant differential operator acting on sections of some $\Gamma$-equivariant Hermitian vector bundle over $X$. We use these data to define Toeplitz operators with symbols in the transformation group $C^{*}$-algebra $C(X) \rtimes \Gamma$. If the symbol of such a Toeplitz operator is invertible, then the operator is Fredholm. In the case where $X$ is a spin manifold and $D$ is the Dirac operator, we give a geometric-topological formula for the index.
\end{abstract}

Let $X$ be a smooth compact manifold without boundary, let $V$ be a Hermitian vector bundle over $X$, and suppose $D$ is a first-order elliptic self-adjoint differential operator acting on sections of $V$. Let $P$ be the positive spectral projection of $D$. Then $P$ is an order zero pseudodifferential operator, and it follows from standard facts about pseudodifferential operators on compact manifolds that given a smooth function $f$ on $X$, the pointwise multiplication operator $M_{f}$ acting on squareintegrable sections $L^{2}(V)$ of $V$ commutes with $P$ modulo the ideal of compact operators. From this fact it is easy to show that if $f$ is invertible, then the Toeplitz operator $P M_{f}: \operatorname{Ran} P \rightarrow \operatorname{Ran} P$ is a Fredholm operator. Furthermore, the index of $P M_{f}$ can be computed using the Atiyah-Singer Index Theorem; see [Baum and Douglas 1982].

Now suppose that a discrete group $\Gamma$ acts smoothly on both $X$ and $V$ in a compatible way, and suppose that $D$ commutes with the action of $\Gamma$ on sections of $V$. Then $P$ also commutes with this action. In addition, there is a natural action $\rho$ of the transformation group $C^{*}$-algebra $C(X) \rtimes \Gamma$ on $L^{2}(V)$, and $P$ commutes with the elements of $C(X) \rtimes \Gamma$ modulo the compacts. Therefore, whenever $F \in C(X) \rtimes \Gamma$ is invertible, $T_{F}:=P \rho(F)$ is a Fredholm operator, and it is natural to ask what the index of this operator is.

Let $X$ be an odd-dimensional oriented spin manifold and let $\Gamma$ be a finite group acting on $X$ by isometries that preserve the orientation and spin structure of $X$. In [Park 2002], the case of free actions was considered; in this paper we consider

MSC2000: primary 47A53; secondary 19K56, 47B35, 46L87.

Keywords: Toeplitz operators, index theory, transformation group $C^{*}$-algebras. 
general actions. We use the Lefschetz theorem in [Fang 2005] to prove a theorem that computes the Fredholm index of $T_{F}$ in terms of the geometry and topology of $X$ and a "Chern character" form explicitly constructed from $F$.

We begin by more precisely defining the objects under discussion. Let $X$ be a smooth compact manifold, and let $\Gamma$ be a discrete group acting smoothly on $X$ from the right. Let $V$ be a $\Gamma$-equivariant complex vector bundle over $X$, and equip $V$ with a $\Gamma$-invariant Hermitian structure. Then $\Gamma$ acts on the left on both the smooth sections $C^{\infty}(V)$ and the square-integrable sections $L^{2}(V)$ of $V$ :

$$
(\gamma \cdot s)(x)=s(x \gamma) \gamma^{-1} .
$$

If $C(X)$ acts on $L^{2}(V)$ by pointwise multiplication, we have a covariant representation of $(C(X), \Gamma)$ on $L^{2}(V)$, and hence a representation $\rho$ of $C(X) \rtimes \Gamma$ on $L^{2}(V)$.

Let $D$ be a first-order, $\Gamma$-equivariant, elliptic self-adjoint differential operator acting on sections of $V$, and let $P=\chi_{[0, \infty)}(D)$ denote the positive spectral projection of $D$; this operator is also $\Gamma$-equivariant. For each $F$ in $C(X) \rtimes \Gamma$, define the Toeplitz operator $T_{F} \in \mathscr{B}\left(L^{2}(V)\right)$ to be $T_{F}=P \rho(F) P+I-P$. More generally, for each positive integer $n$, let $P_{n} \in \mathscr{B}\left(\left(L^{2}(V)\right)^{n}\right)$ be the matrix with $P$ as each diagonal entry and all other entries zero, and let $\rho_{n}$ denote the obvious representation of $\mathrm{M}(n, C(X) \rtimes \Gamma)$ on $\left(L^{2}(V)\right)^{n}$ determined by $\rho$. Then for each $F$ in $\mathrm{M}(n, C(X) \rtimes \Gamma)$, let $T_{F}=P_{n} \rho_{n}(F) P_{n}+I-P_{n}$. We note that while Toeplitz operators are typically defined as operators on the range of $P_{n}$, we have opted to extend our Toeplitz operators to all of $\left(L^{2}(V)\right)^{n}$ in a way that does not affect their index theory.

Proposition 1. If $F$ is in $\operatorname{GL}(n, C(X) \rtimes \Gamma)$, then $T_{F}$ is Fredholm.

Proof. It suffices to show that $P$ commutes with elements of $C(X) \rtimes \Gamma$ modulo the compacts, for then it follows easily that $T_{F} T_{F^{-1}}=I=T_{F^{-1}} T_{F} \bmod \mathscr{K}\left(\left(L^{2}(V)\right)^{n}\right)$. The operator $P$ is $\Gamma$-equivariant and therefore commutes with $\rho(\gamma)$ for each $\gamma$ in $\Gamma$. On the other hand, for all $f$ in $C(X)$, the commutator $[P, \rho(f)]$ is in $\mathscr{K}\left(L^{2}(V)\right)$, by [Baum and Douglas 1982, Lemma 2.10]. These elements are dense in $C(X) \rtimes \Gamma$, so the desired conclusion follows.

Our goal is to find a geometric-topological formula for the index of $T_{F}$ that can be computed directly from $F$. To this end, we will show that the Fredholm index of $T_{F}$ is equal to the $\Gamma$-invariant index of a certain $\Gamma$-equivariant operator.

Definition 2. Let $V$ be a $\Gamma$-equivariant complex vector bundle over $X$, and let $R$ be a $\Gamma$-equivariant elliptic pseudodifferential operator acting on sections of $V$. The $\Gamma$-invariant index of $R$ is denoted by $\operatorname{Ind}_{\Gamma-i n v}(R)$, and is the dimension of the $\Gamma$-invariant subspace of ker $R$ minus the dimension of the $\Gamma$-invariant subspace of ker $R^{*}$. 
Proposition 3. Let $R$ be a $\Gamma$-equivariant elliptic operator. Then

$$
\operatorname{Ind}_{\Gamma-i n v}(R)=\frac{1}{|\Gamma|} \sum_{\gamma \in \Gamma} \operatorname{Ind}_{\gamma}(R),
$$

where $\operatorname{Ind}_{\gamma}(R)$ is the trace of the $\Gamma$-equivariant index of $R$ evaluated at $\gamma$.

Proof. Let $\sigma$ be a representation of $\Gamma$ on a finite-dimensional complex vector space $W$, and decompose $\sigma$ as

$$
\sigma=n_{0} \mathbf{1}+\sum_{i=1}^{k} n_{i} \sigma_{i}
$$

where the $\sigma_{i}$ are irreducible and distinct, and 1 denotes the trivial representation. Let $W^{\Gamma}$ be the subspace of $W$ that is fixed by $\Gamma$. The fact that each of the $\sigma_{i}$ fixes only 0 implies that $n_{0}=\operatorname{dim} W^{\Gamma}$. Then, by [Serre 1977, Exercise 2.5],

$$
\operatorname{dim} W^{\Gamma}=\frac{1}{|\Gamma|} \sum_{\gamma \in \Gamma} \chi(\gamma)=\frac{1}{|\Gamma|} \sum_{\gamma \in \Gamma} \operatorname{Tr}(\sigma(\gamma)),
$$

whence the result follows.

For each natural number $n$, let $\operatorname{Map}(\Gamma, \mathrm{M}(n, C(X)))$ denote the $C^{*}$-algebra of all functions from the group $\Gamma$ to $\mathrm{M}(n, C(X))$. The algebra $\mathrm{M}(n, C(X))$ acts on $\operatorname{Map}(\Gamma, \mathrm{M}(n, C(X)))$ via pointwise multiplication; let $\mathscr{L}(\operatorname{Map}(\Gamma, \mathrm{M}(n, C(X))))$ be the algebra of $\mathrm{M}(n, C(X))$-linear maps on $\operatorname{Map}(\Gamma, \mathrm{M}(n, C(X)))$. We define a homomorphism

$$
\mu: \mathrm{M}(n, C(X)) \rtimes \Gamma \longrightarrow \mathscr{L}(\operatorname{Map}(\Gamma, \mathrm{M}(n, C(X))))
$$

as follows: for all $\psi$ in $\operatorname{Map}(\Gamma, \mathrm{M}(n, C(X)))$, all functions $f$ in $\mathrm{M}(n, C(X))$, and all elements $\alpha$ and $\gamma$ in $\Gamma$, set $(\mu(f) \psi)(\alpha)=\mu(\alpha \cdot f) \psi(\alpha)$ and $(\mu(\gamma))(\alpha)=$ $\psi\left(\gamma^{-1} \alpha\right)$, and then extend $\mu$ to all of $\mathrm{M}(n, C(X)) \rtimes \Gamma$ by stipulating that $\mu$ be an algebra homomorphism. If $\mathrm{M}(n, C(X))$ acts on $\left(L^{2}(V)\right)^{n}$ by pointwise multiplication and if $\lambda$ denotes the left regular representation of $\mathrm{M}(n, C(X))$ on the Hilbert space $\operatorname{Map}\left(\Gamma,\left(L^{2}(V)\right)^{n}\right)$, then $\lambda(F)$ is matrix multiplication by $\mu(F)$ for every $F$ in $\mathrm{M}(n, C(X) \rtimes \Gamma$.

The group $\Gamma$ acts on $\operatorname{Map}\left(\Gamma,\left(L^{2}(V)\right)^{n}\right)$ by the formula

$$
(\gamma \cdot \psi)(\alpha)=\gamma \cdot\left(\psi\left(\gamma^{-1} \alpha\right)\right),
$$

and the subspace $\operatorname{Map}\left(\Gamma,\left(L^{2}(V)\right)^{n}\right)^{\Gamma}$ of elements fixed by $\Gamma$ contains precisely the $\psi$ for which $\gamma \cdot(\psi(e))=\psi(\gamma)$ for all $\gamma$ in $\Gamma$; here $e$ denotes the identity element of $\Gamma$. Thus the elements of $\operatorname{Map}\left(\Gamma,\left(L^{2}(V)\right)^{n}\right)^{\Gamma}$ are determined by their value at $e$. Conversely, specifying a value at $e$ uniquely determines an element of $\operatorname{Map}\left(\Gamma,\left(L^{2}(V)\right)^{n}\right)^{\Gamma}$. 
Define $U:\left(L^{2}(V)\right)^{n} \rightarrow \operatorname{Map}\left(\Gamma,\left(L^{2}(V)\right)^{n}\right)^{\Gamma}$ as

$$
\left(U\left(s_{1}, s_{2}, \ldots, s_{n}\right)\right)(\alpha)=\frac{1}{\sqrt{|\Gamma|}}\left(\alpha \cdot s_{1}, \alpha \cdot s_{2}, \ldots, \alpha \cdot s_{n}\right) .
$$

Because the inner product on $L^{2}(V)$ has been chosen to be $\Gamma$-invariant, $U$ is a unitary operator, and $U^{*} \psi=\sqrt{|\Gamma|} \psi(e)$ for every $\psi$.

Define $\widetilde{\mathrm{D}}$ on $\operatorname{Map}\left(\Gamma,\left(L^{2}(V)\right)^{n}\right)$ by the formula $(\widetilde{\mathrm{D}} \psi)(\alpha)=D(\psi(\alpha))$. Let $\widetilde{\mathrm{P}}$ be the positive spectral projection of $\widetilde{\mathrm{D}}$, and for each $F$ in $\mathrm{M}(n, C(X) \rtimes \Gamma)$, define

$$
\widetilde{\mathrm{T}}_{F}=\widetilde{\mathrm{P}} \lambda(F) \widetilde{\mathrm{P}}+I-\widetilde{\mathrm{P}} .
$$

We can express the Fredholm index of Toeplitz operators $T_{F}$ in terms of the $\widetilde{\mathrm{T}}_{F}$ :

Proposition 4. For every $F$ in $\operatorname{GL}(n, C(X) \rtimes \Gamma)$,

$$
\text { Ind } T_{F}=\frac{1}{|\Gamma|} \sum_{\gamma \in \Gamma} \operatorname{Ind}_{\gamma} \widetilde{\mathrm{T}}_{F} .
$$

Proof. For each $F$, the operator $\widetilde{\mathrm{T}}_{F}$ is $\Gamma$-equivariant with symbol $\mu(F)$. Furthermore, when $\widetilde{\mathrm{T}}_{F}$ is restricted to the Hilbert space $\operatorname{Map}\left(\Gamma,\left(L^{2}(V)\right)^{n}\right)^{\Gamma}$, we have $\widetilde{\mathrm{T}}_{F}=U T_{F} U^{*}$. Thus the result follows immediately from Propositions 1 and 3.

For the remainder of the paper, we will assume that $X$ is a $(2 m+1)$-dimensional spin manifold with spinor bundle $S$ and Dirac operator $D$, and that $\Gamma$ acts on $X$ by orientation-preserving isometries that preserve the spin structure. In this case, we can combine Proposition 4 with the index theorem in [Fang 2005] to get a geometric-topological index formula for the index of $T_{F}$.

Theorem 5. For each $\gamma$ in $\Gamma$, let $X_{1}^{\gamma}, X_{2}^{\gamma}, \ldots, X_{K_{\gamma}}^{\gamma}$ be the connected components of the fixed point set of $\gamma$, and for each $1 \leq k \leq K_{\gamma}$, let $N X_{k}^{\gamma}$ denote the normal bundle of $X_{k}^{\gamma}$ in $X$. Then for all $F$ in $\operatorname{GL}\left(n, C^{\infty}(X) \rtimes \Gamma\right)$,

$$
\text { Ind } T_{F}=\frac{1}{|\Gamma|} \sum_{\gamma \in \Gamma} \sum_{k=1}^{K_{\gamma}}\left(\frac{-i}{2 \pi}\right)^{\left(1+\operatorname{dim} X_{k}^{\gamma}\right) / 2} \int_{X_{k}^{\gamma}} \widehat{A}\left(X_{k}^{\gamma}\right) \operatorname{ch}(\mu(F)) \Lambda^{-1},
$$

where

$$
\left.\operatorname{ch}(\mu(F))=\sum_{k=0}^{\infty}(-1)^{k} \frac{k !}{(2 k+1) !} \operatorname{Tr}\left((\mu(F))^{-1} d \mu(F)\right)^{2 k+1}\right)
$$

and

$$
\Lambda=\operatorname{Pf}\left(2 \sin \left(\frac{i}{2}\left(R\left(N X_{k}^{\gamma}\right)+\ln J\left(X_{k}^{\gamma}\right)\right)\right)\right) ;
$$

here $\mathrm{Pf}$ is the Pfaffian, $R\left(N X_{k}^{\gamma}\right)$ is the curvature of $N X_{k}^{\gamma}$, and $J\left(X_{k}^{\gamma}\right)$ is the Jacobian matrix of the action of $\gamma$ on $N X_{k}^{\gamma}$. 
For this theorem to be useful to us, we need to be able to express $\operatorname{ch}(\mu(F))$ in terms of $F$.

The action of $F$ on $X$ induces an action on the algebra $\Omega^{*}(X)$ of smooth differential forms, and we can extend our map $\mu$ to an algebra homomorphism

$$
\mu: \mathrm{M}\left(n, \Omega^{*}(X)\right) \rtimes \Gamma \longrightarrow \mathscr{L}\left(\operatorname{Map}\left(\Gamma, \mathrm{M}\left(n, \Omega^{*}(X)\right)\right)\right) .
$$

Take $\sum_{\gamma \in \Gamma} \omega_{\gamma} \gamma$ in $\mathrm{M}\left(n, \Omega^{*}(X)\right) \rtimes \Gamma$. Then for all $\psi$ in $\operatorname{Map}\left(\Gamma, \mathrm{M}\left(n, \Omega^{*}(X)\right)\right)$,

$$
\left(\mu\left(\sum_{\gamma \in \Gamma} \omega_{\gamma} \gamma\right) \psi\right)(\alpha)=\sum_{\gamma \in \Gamma}\left(\alpha \cdot \omega_{\gamma}\right) \psi(\alpha \gamma) .
$$

For any element $B$ of $\mathscr{L}\left(\operatorname{Map}\left(\Gamma, \mathrm{M}\left(n, \Omega^{*}(X)\right)\right)\right)$, its trace is computed by the formula

$$
\operatorname{Tr}(B)=\sum_{\alpha \in \Gamma}\left(B \delta_{\alpha}\right)(\alpha)
$$

where $\delta_{\alpha}$ is the constant function 1 when evaluated at $\alpha \in \Gamma$, and is otherwise zero. Thus

$$
\begin{aligned}
\operatorname{Tr}\left(\mu\left(\sum_{\gamma \in \Gamma} \omega_{\gamma} \gamma\right)\right) & =\sum_{\alpha, \gamma \in \Gamma}\left(\mu\left(\omega_{\gamma} \gamma\right) \delta_{\alpha}\right)(\alpha) \\
& =\sum_{\alpha, \gamma \in \Gamma}\left(\alpha \cdot \omega_{\gamma}\right) \delta_{\alpha}(\alpha \gamma) \\
& =\sum_{\alpha \in \Gamma} \alpha \cdot \omega_{e} .
\end{aligned}
$$

Definition 6. Let $v: \mathrm{M}\left(n, \Omega^{*}(X)\right) \rtimes \Gamma \rightarrow \Omega^{*}(X)$ be given by the formula

$$
v\left(\sum_{\gamma \in \Gamma} \omega_{\gamma} \gamma\right)=\sum_{\alpha \in \Gamma} \alpha \cdot \omega_{e},
$$

and for all $F$ in $\operatorname{GL}\left(n, C^{\infty}(X) \rtimes \Gamma\right)$, define

$$
\widehat{\operatorname{ch}}(F)=\sum_{k=0}^{\infty}(-1)^{k} \frac{k !}{(2 k+1) !} v\left(\left(F^{-1} d F\right)^{2 k+1}\right),
$$

where the exterior derivative $d$ is extended to $\mathrm{M}\left(n, \Omega^{*}(X)\right) \rtimes \Gamma$ by applying $d$ entrywise in $\mathrm{M}\left(n, C^{\infty}(X)\right)$ and setting

$$
d\left(\sum_{\gamma \in \Gamma} \omega_{\gamma} \gamma\right)=\sum_{\gamma \in \Gamma}\left(d \omega_{\gamma}\right) \gamma
$$

Combining Theorem 5 and Definition 6, we have: 
Theorem 7. For all $F$ in $\operatorname{GL}\left(n, C^{\infty}(X) \rtimes \Gamma\right)$,

$$
\text { Ind } T_{F}=\frac{1}{|\Gamma|} \sum_{\gamma \in \Gamma} \sum_{k=1}^{K_{\gamma}}\left(\frac{-i}{2 \pi}\right)^{\left(1+\operatorname{dim} X_{k}^{\gamma}\right) / 2} \int_{X_{k}^{\gamma}} \widehat{A}\left(X_{k}^{\gamma}\right) \widehat{\operatorname{ch}}(F) \Lambda^{-1} .
$$

This formula looks rather daunting, but in many cases it simplifies considerably.

Example 8. Let $\mathrm{SO}(2 m+2)$ act on $\mathbb{R}^{2 m+2}$ in the usual way, let $\Gamma$ be a finite subgroup of $\mathrm{SO}(2 m+2)$, and let $S^{2 m+1}$ be the unit sphere in $\mathbb{R}^{2 m+2}$. The action of $\Gamma$ on $\mathbb{R}^{2 m+2}$ restricts to an action on $S^{2 m+1}$, and because spheres have unique spin structures [Lawson and Michelsohn 1989], the action of $\Gamma$ on $S^{2 m+1}$ trivially preserves the spin structure. Now, each $\gamma$ in $\Gamma$ fixes a subspace of $\mathbb{R}^{2 m+2}$, and so the fixed point set $X^{\gamma}$ of $\gamma$ acting on $S^{2 m+1}$ is an equatorial sphere, and in particular, the fixed point set is connected. Furthermore, all spheres have stably trivial tangent bundles, so $\widehat{A}\left(X^{\gamma}\right)=1$ for all $\gamma$ in $\Gamma$. Finally, a straightforward computation shows that the normal bundle of an equatorial sphere has curvature zero, and so

$$
\text { Ind } T_{F}=\frac{1}{|\Gamma|} \sum_{\gamma \in \Gamma}\left(\frac{-i}{2 \pi}\right)^{\left(1+\operatorname{dim} X^{\gamma}\right) / 2} \int_{X^{\gamma}} \widehat{\operatorname{ch}}(F)\left(\operatorname{Pf}\left(2 \sin \left(\frac{1}{2} \ln J\left(X^{\gamma}\right)\right)\right)\right)^{-1} .
$$

Given $\gamma$ in $\Gamma$, there exists an orthonormal frame of $N X^{\gamma}$ for which the action of $\gamma$ on $N X^{\gamma}$ decomposes into blocks

$$
\left(\begin{array}{rr}
\cos \theta_{j}^{\gamma} & \sin \theta_{j}^{\gamma} \\
-\sin \theta_{j}^{\gamma} & \cos \theta_{j}^{\gamma}
\end{array}\right)
$$

with $0<\theta_{j}^{\gamma}<2 \pi$ and $j=1,2, \ldots, L^{\gamma}=m-\frac{1}{2}\left(\operatorname{dim} X^{\gamma}+1\right)$ (see [Lawson and Michelsohn 1989]). Incorporating this into our formula we obtain

$$
\text { Ind } T_{F}=\frac{1}{|\Gamma|} \sum_{\gamma \in \Gamma}\left(\frac{-i}{2 \pi}\right)^{\left(1+\operatorname{dim} X^{\gamma}\right) / 2} \frac{1}{\sin \left(\theta_{1}^{\gamma} / 2\right) \cdots \sin \left(\theta_{L^{\gamma}}^{\gamma} / 2\right)} \int_{X^{\gamma}} \widehat{\operatorname{ch}}(F) .
$$

\section{Acknowledgment}

The author thanks Ken Richardson for helpful discussions.

\section{References}

[Baum and Douglas 1982] P. Baum and R. G. Douglas, "Toeplitz operators and Poincaré duality", pp. 137-166 in Toeplitz centennial (Tel Aviv, 1981), edited by I. Gohberg, Operator Theory: Adv. Appl. 4, Birkhäuser, Basel, 1982. MR 84m:58139 Zbl 0517.55001

[Fang 2005] H. Fang, "Equivariant spectral flow and a Lefschetz theorem on odd-dimensional spin manifolds", Pac. J. Math. 220 (2005), 299-312. 
[Lawson and Michelsohn 1989] H. B. Lawson, Jr. and M.-L. Michelsohn, Spin geometry, Princeton Mathematical Series 38, Princeton University Press, Princeton, NJ, 1989. MR 91g:53001 Zbl 0688.57001

[Park 2002] E. Park, "The index of Toeplitz operators on free transformation group $C^{*}$-algebras", Bull. London Math. Soc. 34:1 (2002), 84-90. MR 2002m:58034 Zbl 1030.58016

[Serre 1977] J.-P. Serre, Linear representations of finite groups, Graduate Texts in Mathematics 42, Springer, New York, 1977. MR 56 \#8675 Zbl 0355.20006

Received December 10, 2003. Revised July 22, 2005.

\section{EFTON PARK}

DEPARTMENT OF MATHEMATICS

TEXas Christian UnIVERSITY

BOX 298900

FORT WORTH, TX 76129

e.park@tcu.edu 